\title{
Outcomes of ureteroscopy and internal ureteral stent for pregnancy with urolithiasis: a systematic review and meta-analysis
}

\author{
Xingwei Jin ${ }^{1}$, Boke $\mathrm{Liu}^{1}$, Yunqi Xiong${ }^{2}$, Weichao $\mathrm{Tu}^{1}$, Yuan Shao ${ }^{1}$, Lin Zhang ${ }^{3}$, and Dawei \\ Wang ${ }^{1}$ \\ ${ }^{1}$ Shanghai Jiao Tong University Medical School Affiliated Ruijin Hospital \\ ${ }^{2}$ Shanghai TCM College Affiliated Shu Guang Hospital \\ ${ }^{3}$ School of Population Medicine and Public Health, Chinese Academy of Medical Science \& \\ Peking Union Medical College
}

February 5, 2021

\begin{abstract}
Objectives To investigate the outcomes of internal ureteral stent versus ureteroscopy for pregnant women with urolithiasis. Data Sources Relevant studies published from January 1980 to April 2020 were identified through a systematic literature search in MEDLINE, EMBASE, Web of Science and Cochrane Library. Study Eligibility Criteria Total of 453 studies were initially identified. Pregnant women in any pregnancy stages who underwent D-J stent insertion only or ureteroscopy operation were included. The number of related participants in each group of study should be more than 10. This systematic review has been registered on PROSPERO (CRD42020195607). Results A total of 25 studies were identified with 131 cases serial stenting and 789 cases URS. The pooled operation success rate was $97 \%$ for D-J stent insertion, and $99 \%$ for URS. For internal ureteral stent therapy, normal fertility outcome rate was $99 \%$, but the pooled incidence of complications was about $45 \%$. For the URS group, normal fertility outcome rate was $99 \%$, and the pooled incidence of complications was about $1 \%$. However, the pooled premature and abortion incidence rate of two groups were the same as less than $1 \%$, and same in serious complication incidence rate. Conclusions Although internal ureteral stent may cause more slight complications, ureteroscopy operation and internal ureteral stent showed less side effective on fertility results. Evidence suggests that URS therapy have greater advantage for pregnancy with urinary stones when the condition permits. As it is proved safe and effective, internal ureteral stent could be considered at emergency or other special situations.
\end{abstract}

\section{Hosted file}

maintext.pdf available at https://authorea.com/users/393980/articles/507469-outcomes-ofureteroscopy-and-internal-ureteral-stent-for-pregnancy-with-urolithiasis-a-systematicreview-and-meta-analysis 
Figure 2. Meta-analysis about operation success rate in D-J stent therapy group and URS group.

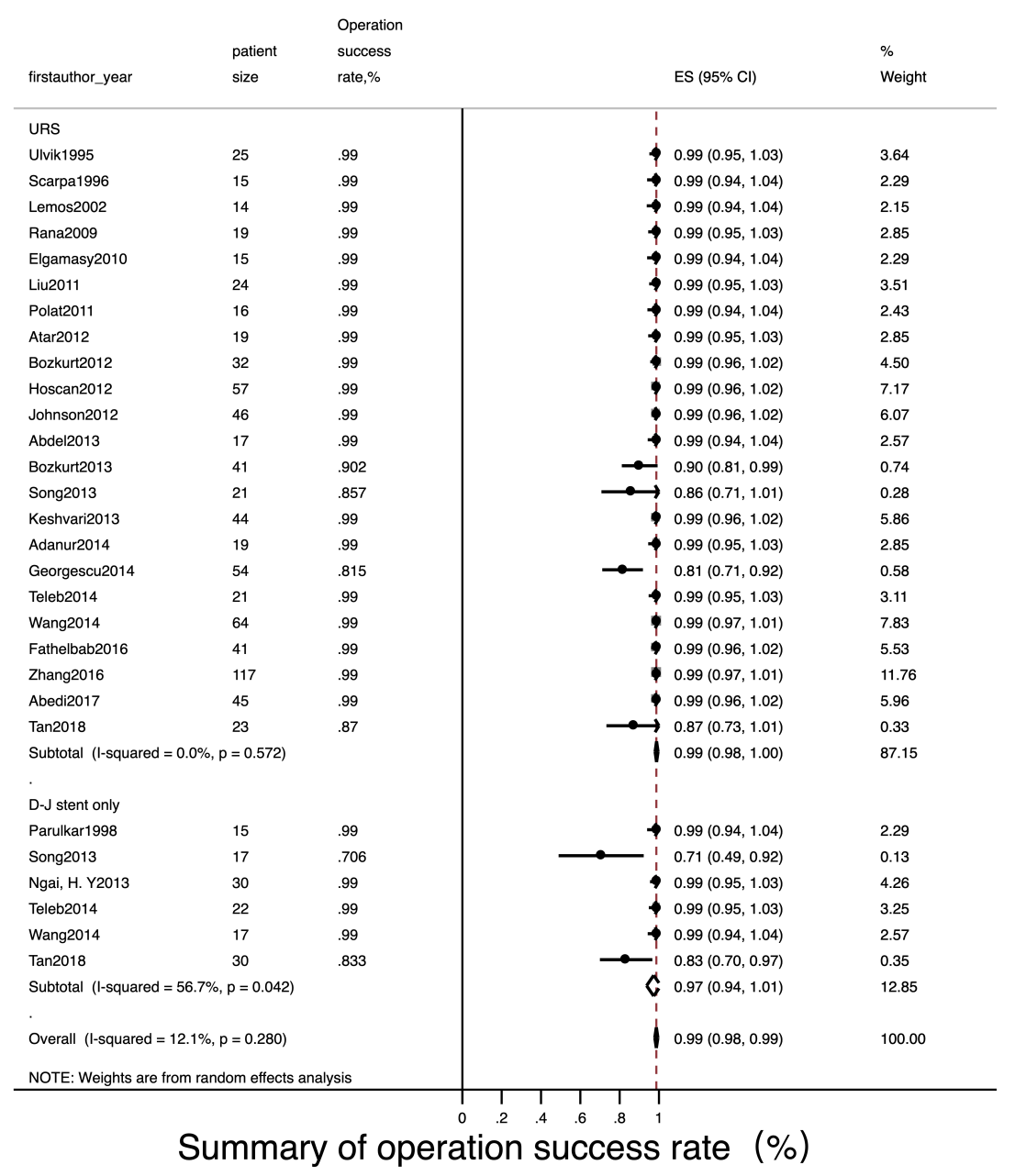


Figure 3. Meta-analysis about normal fertility outcome in D-J stent therapy group and URS group.

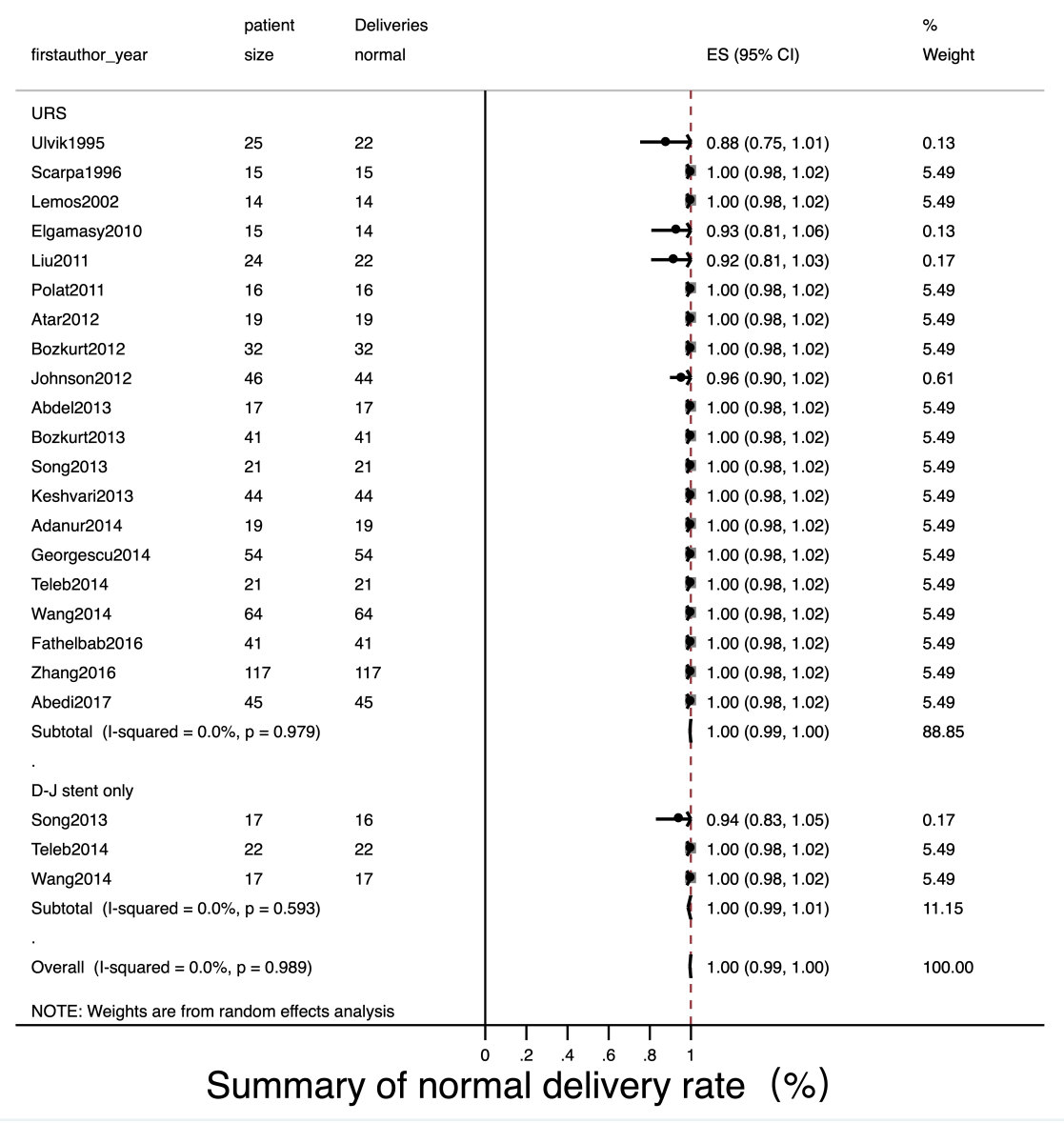


Figure 4. Meta-analysis about adverse pregnant outcome (premature and abortion) in D-J stent therapy group and URS group.

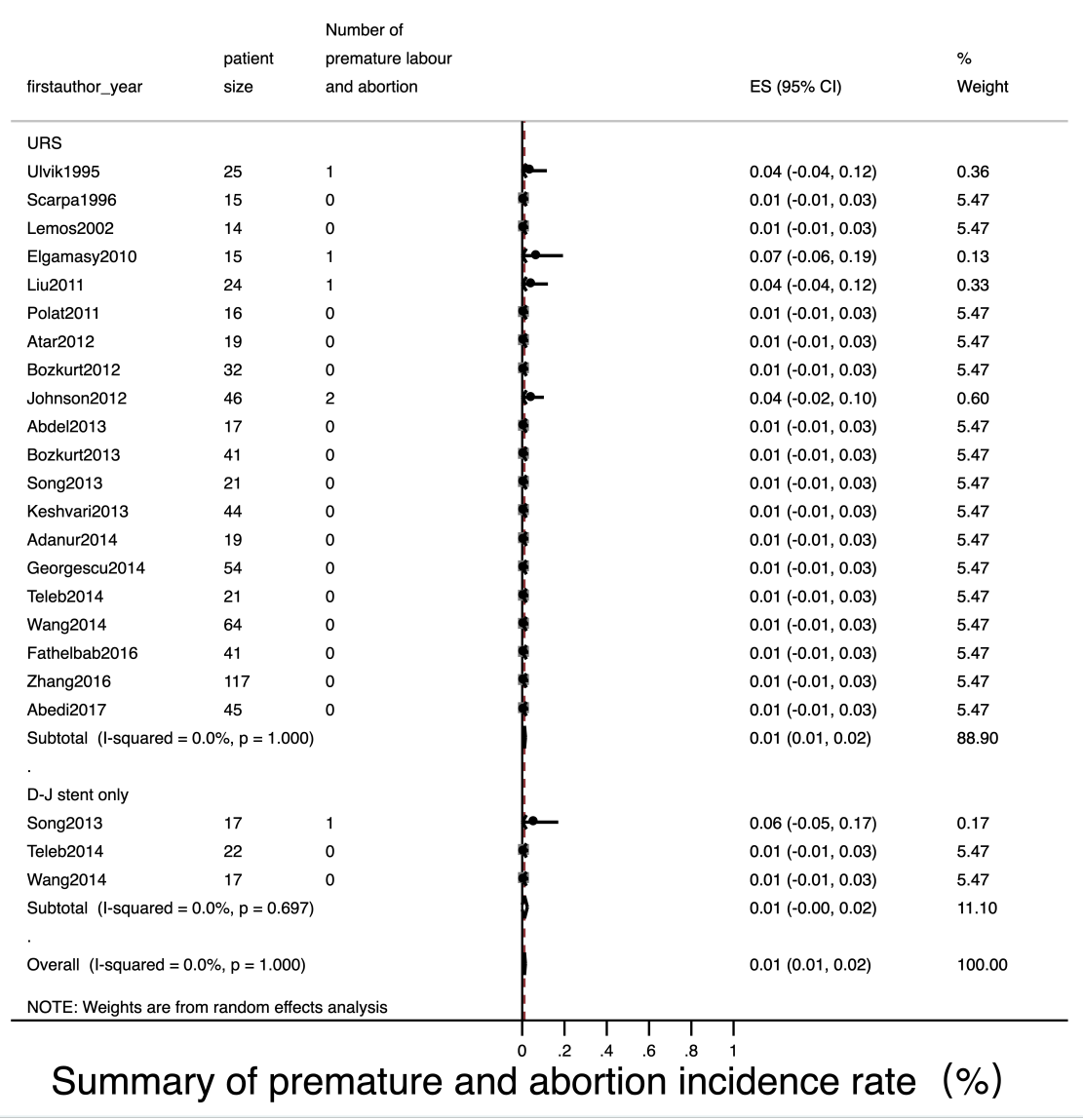


Figure 5. Meta-analysis about overall complications in D-J stent therapy group and URS group.

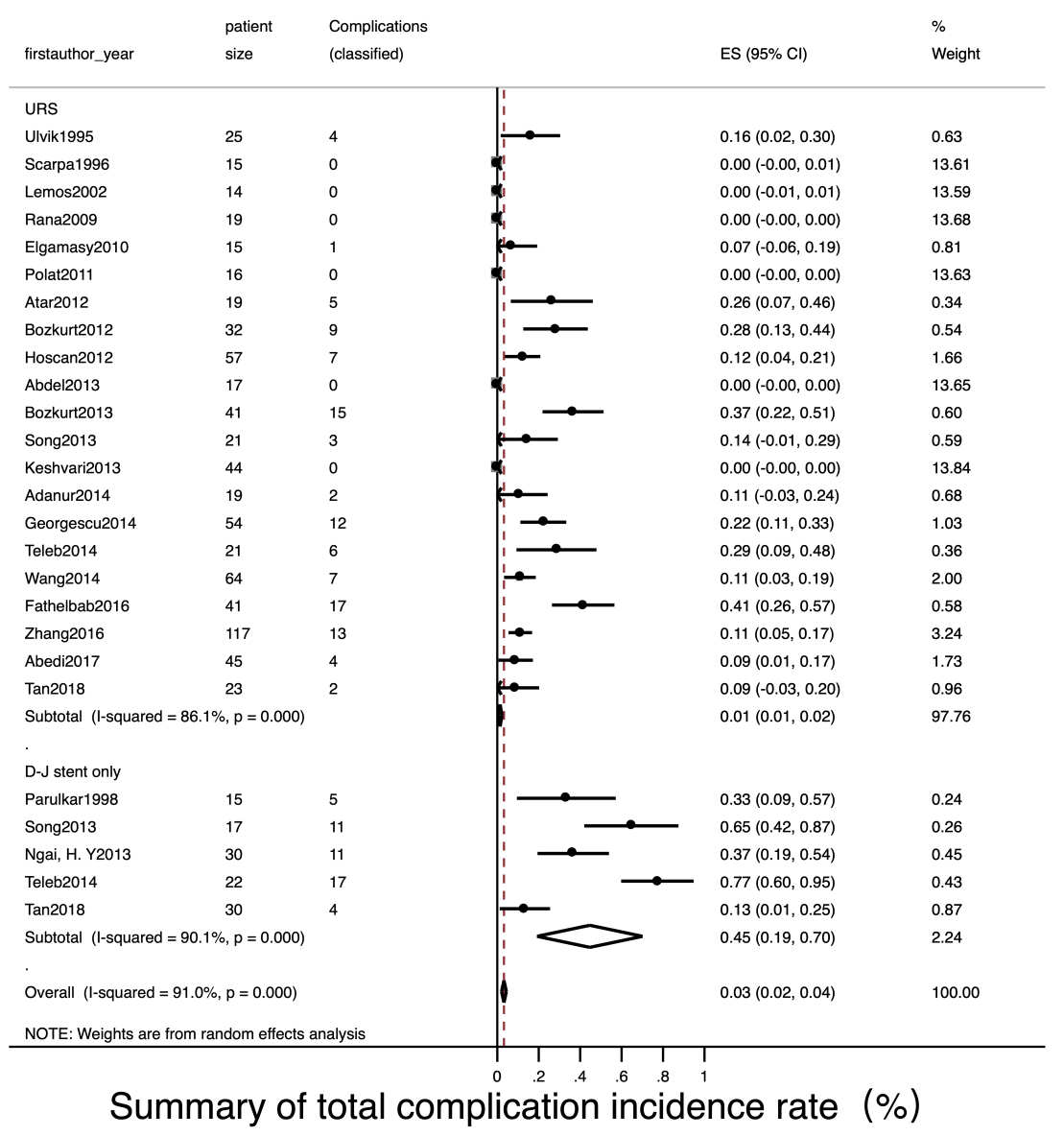


Figure 6. Meta-analysis about Clavien-Dindo III-V complications in D-J stent therapy group and URS group.

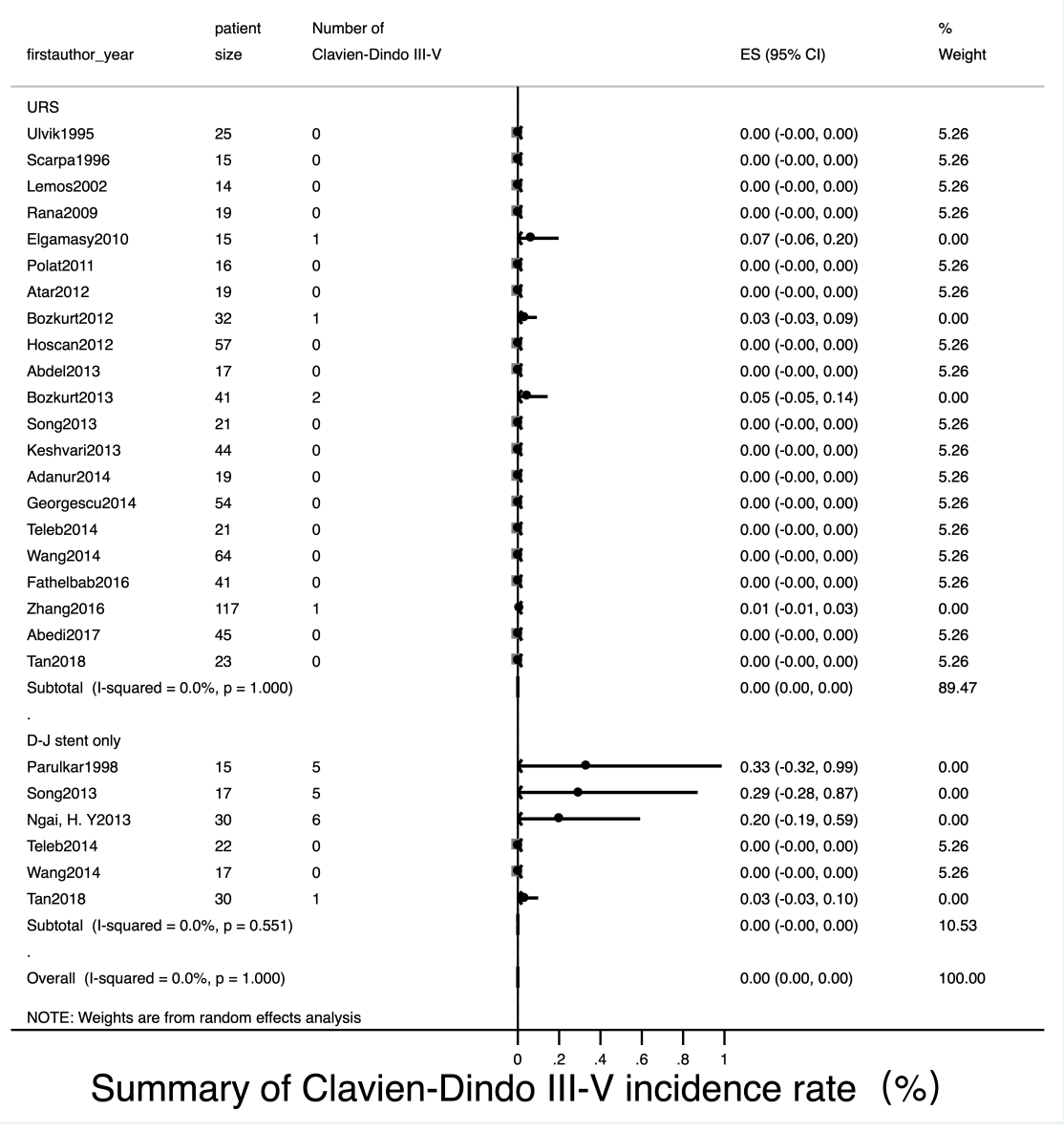


Figure S1. Funnel Plot for Publication Bias

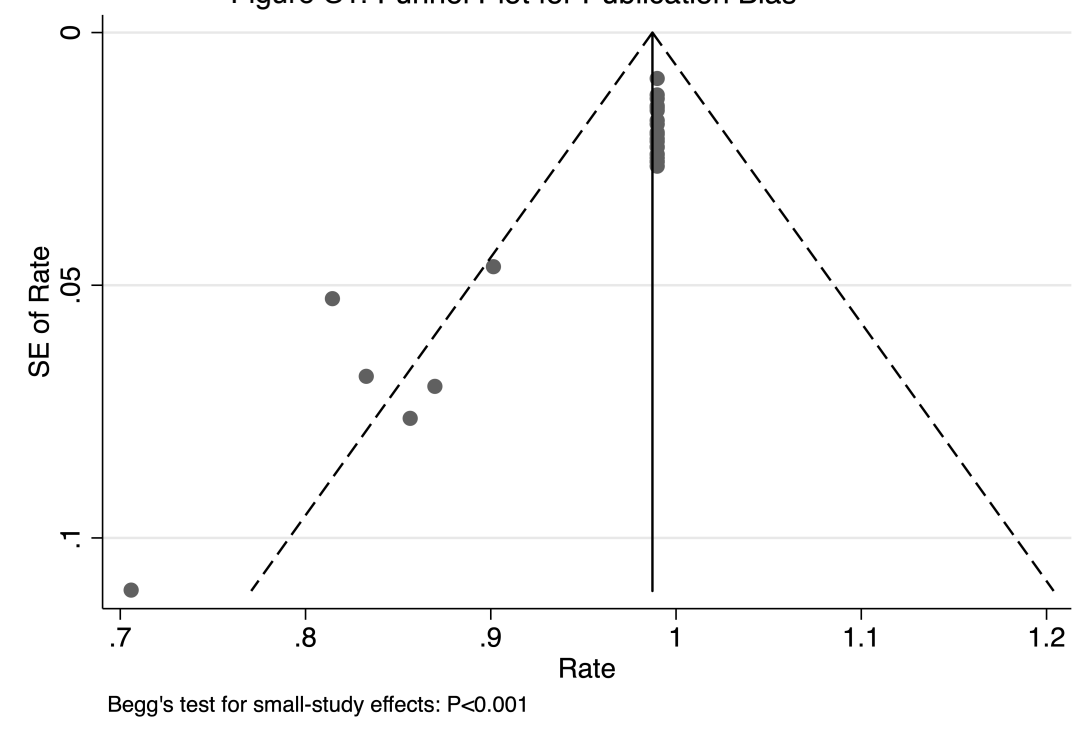


Figure 1. PRISMA flow diagram of study selection for meta-analysis

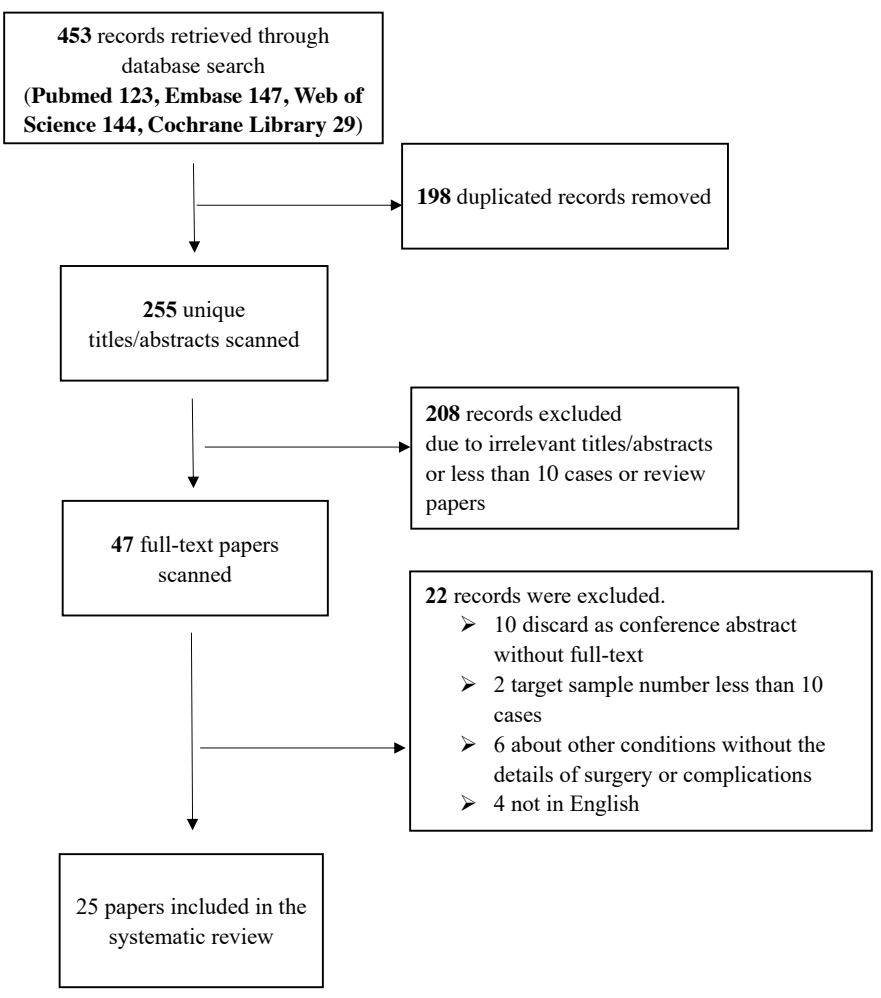

